\title{
Frontières
}

\section{Bonne mort et male mort au Japon et dans d'autres sociétés}

\section{Diane Laflamme}

Volume 20, numéro 1, automne 2007

La « bonne mort»

URI : https://id.erudit.org/iderudit/017961ar

DOI : https://doi.org/10.7202/017961ar

Aller au sommaire du numéro

Éditeur(s)

Université du Québec à Montréal

ISSN

1180-3479 (imprimé)

1916-0976 (numérique)

Découvrir la revue

Citer ce document

Laflamme, D. (2007). Bonne mort et male mort au Japon et dans d'autres sociétés. Frontières, 20(1), 108-111. https://doi.org/10.7202/017961ar d'utilisation que vous pouvez consulter en ligne.

https://apropos.erudit.org/fr/usagers/politique-dutilisation/ 


\title{
Bonne mort et male mort au Japon et dans d'autres sociétés
}

\author{
Diane Laflamme, Ph. D., \\ professeure associée, \\ Département de sciences des religions, UQAM.
}

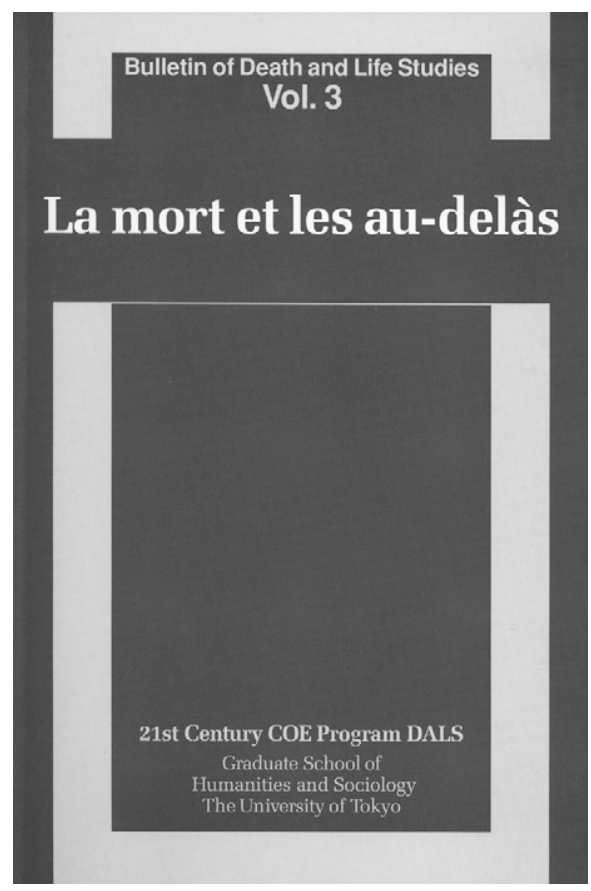

UNIVERSITÉ DE TOKYO

La mort et les au-delàs

Bulletin of Death and Life Study,

vol. 3

Institut des sciences humaines et sociales, 21st Century COE Program DALS, Tokyo, 2007, 224 p.

(textes en français avec résumés en anglais)

Nous apprenons grâce à ces deux publications que l'Institut des sciences humaines et sociales de l'Université de Tokyo a établi un Centre d'excellence (Centre of Excellence, COE) dont les travaux portent sur la "Construction des études de la mort et de la vie concernant la culture et la valeur de la vie»(Death and Life Studies, DALS). Le Programme du XXI ${ }^{\mathrm{e}}$ siècle COE, DALS, placé depuis 2002 sous la direction du professeur Susumu Shimazono, se compose de quatre sections: Repenser les études de la mort et de la vie dans la perspective de la philosophie pratique, Images et perspectives de la mort et de la vie, Civilisation et valeurs concernant les perspectives de la mort et de la vie, et Recherche sur la perspective des êtres humains comme expression des activités de la vie (I, p. 223-224). Les professeurs et maîtres de conférences proviennent de divers horizons disciplinaires: études des religions, philosophie, éthique, sociologie, psychologie, sciences de santé, histoire et philosophie de l'éducation, littérature, linguistique, histoire de l'art, études des ressources culturelles et archéologie. Un regroupement de disciplines qui ferait envie à plus d'un chercheur québécois qui œuvre à promouvoir chez nous l'interdisciplinarité!

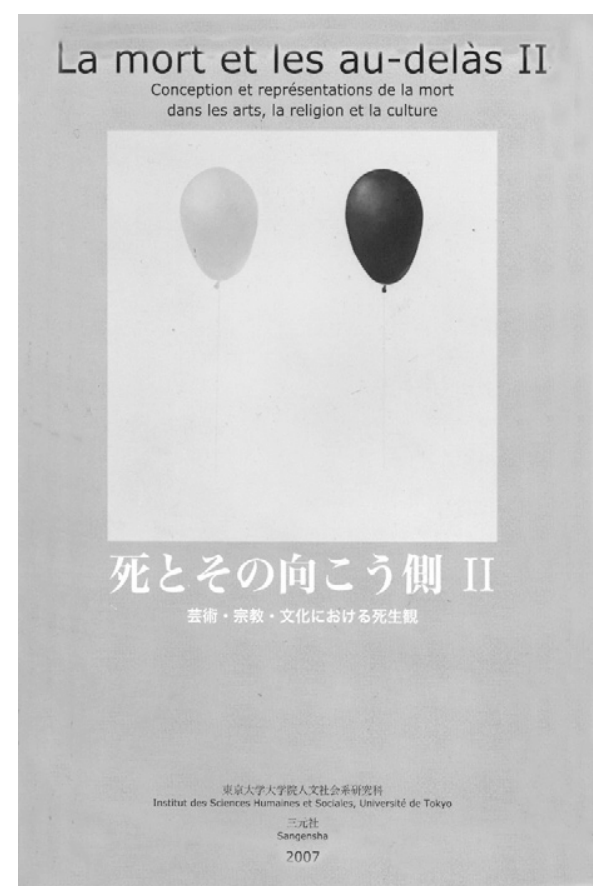

UNIVERSITÉ DE TOKYO

\section{La mort et les au-delàs II}

Conception et représentations de la mort dans les arts, la religion et la culture

Institut des sciences humaines et sociales, 21st Century COE Program DALS, Tokyo, Sangensha Publishers Inc., 2007, 431 p.

(textes en japonais avec traduction en français ou en anglais)

Des chercheurs de ce programme se sont joints à l'équipe du professeur Jean-Pierre Albert du Centre d'anthropologie de Toulouse pour permettre la tenue de deux colloques sur le thème de la mort et des au-delàs, d'abord à Tokyo, en février 2006, puis à Toulouse, en France, en octobre 2006. Les actes de ces colloques ont été publiés dans deux volumes contenant des textes en japonais, en français et en anglais (seul le volume II de La mort et les au-delàs contient une première partie où tous les textes sont en japonais et une seconde partie où ces mêmes textes sont présentés la plupart en français et quelques-uns en anglais). On ne peut que se réjouir d'avoir ainsi accès à des résultats de recherches récentes portant sur la conception de la mort et de l'au-delà dans la culture japonaise ancienne et contemporaine: en tout, on peut lire quinze textes portant sur le Japon (treize en français et deux en anglais). S'ajoutent à cela, grâce à l'approche comparative qui guidait les organisateurs de ces colloques, des textes sur la mort et l'au-delà en Europe (8 articles), en Asie (4 articles) et en Amérique du Sud (1 article). Le professeur Shimazono signale d'ailleurs, dans l'avant-propos du premier volume, toute la richesse que l'approche comparative et l'apport interdisciplinaire ont apportée aux échanges et aux débats (I, p. 9-11). 
La formule adoptée pour présenter les textes dans les deux volumes permet difficilement au lecteur d'avoir une vision d'ensemble du riche matériel mis à sa disposition pour chacun des thèmes traités. En effet, les textes nous sont proposés en suivant l'ordre de présentation des conférences publiques et des ateliers lors de chacun des deux colloques et ils sont entrecoupés des comptes rendus des discussions et des débats de synthèse, ce qui rend le repérage plus laborieux. Dans le commentaire qui suit, nous regrouperons les articles selon des catégories différentes de celles figurant dans la table des matières des deux ouvrages, en puisant au besoin dans les deux volumes qui seront identifiés I et II avec l'indication de la page.

Les organisateurs de ces colloques avaient indiqué au nombre de leurs objectifs celui de penser les phénomènes relatifs à la mort et à l'au-delà selon la spécificité de chaque contexte tout en essayant d'en cerner les traits communs (Albert, II, p. 193). Cette approche comparative s'est avérée féconde, comme le confirment les six regroupements proposés ci-après: représentations de l'au-delà ; maladie et guérison, finitude et salut; contacts entre les morts et les vivants; romans, bandes dessinées et théâtre; mort volontaire; et la mort en classe.

\section{LES REPRÉSENTATIONS DE L'AU-DELÀ}

La conception que l'on se fait du monde de l'au-delà a inspiré une riche iconographie et ce thème se prête particulièrement bien à une comparaison entre les représentations propres à diverses cultures. Grâce aux trois articles suivants qui portent sur des œuvres où sont représentés les divers mondes de l'au-delà, le lecteur occidental peut se familiariser avec des chefs d'œuvre du Japon (un mandala, un livre d'images et des sutras enluminés), tout en se référant en parallèle à un retable de l'Europe du Moyen Âge.

Le professeur K. Komine, de l'Université Rikkyo, consacre la première moitié de son article à la problématique de la dualité, en analysant des récits du Konjaku Monogatarishû (XII ${ }^{\mathrm{e}}$ siècle) et du Nihon Ryôki décrivant des expériences de séparation entre le corps et l'âme (tama). La seconde moitié de l'article porte sur deux œuvres iconographiques du XVI ${ }^{\mathrm{e}}$ siècle où l'on trouve une représentation de l'au-delà (I, p. 45-49). Le Jikkai Mandala, peint sur un grand rouleau, présente les Dix univers qui sont formés de Six voies appartenant au domaine des passions et de Quatre saintetés qui représentent le monde sacré. Un bout de ficelle, qui part de chacun des Dix univers, vient s'accrocher à l'idéogramme de la pensée (shin) placé au-dessus du centre du mandala. L'auteur explique qu'il s'agit là d'une idée qui caractérise le bouddhisme Tendai: «chacun des dix univers du mandala constitue un monde indépendant et concret en même temps qu'il englobe l'ensemble des dix univers, eux-mêmes se trouvant dans la pensée de tous les êtres vivants» (I, p. 46). L'auteur signale également qu'il existe des œuvres où l'artiste se sert du mandala pour procéder à un mise en abyme, par exemple le paravent Sumiyoshisha Saireizu Byôbu, ou l'on voit ce même mandala ainsi que la figure d'une nonne qui est en train de l'expliquer à un auditoire et qui pointe avec un bâton sur l'idéogramme de la pensée placé au centre du mandala. On sait jusqu'à quel point les procédés récursifs et la mise en abyme peuvent provoquer le vertige lorsqu'ils sont appliqués à la réflexion intellectuelle ; cette invitation à penser l'au-delà et à observer ce qui se passe dans la démarche même de penser cet au-delà a, effectivement, de quoi provoquer le vertige! Comme le dit l'auteur: "On essaie de voir un monde invisible et on parvient finalement à la voir. Cela veut dire que l'on a déjà modifié la signification de ce monde » (I, p. 47). On ne peut que déplorer que l'article ne soit pas accompagné d'une reproduction du mandala ou du paravent, ce qui nous permettrait d'expérimenter la démarche plus directement. La deuxième œuvre présentée dans l'article illustre un texte qui avait été créé à l'origine pour le théâtre de marionnettes: L'Invasion des enfers par Yoshitsune. L'auteur se réfère à un exemplaire de ce livre d'images qui aurait été exécuté entre la fin du XVII ${ }^{\mathrm{e}}$ siècle et le début du XVIII ${ }^{\mathrm{e}}$ siècle. L'histoire riche en péripéties qui nous est ainsi racontée est une tentative pour visualiser le monde de l'au-delà, encore une fois en présence d'un observateur, cette fois-ci un ascète qui découvre finalement que ce qu'il a pensé se déroulait dans un rêve.

Nous restons dans le Japon ancien avec l'article intitulé Visions $d u$ salut dans la décoration du Heike Nôkyô (Kuroiwa, II, p. 407), qui porte sur une œuvre collective: les trente-trois rouleaux avec enluminures où le Sûtra du Lotus aurait été copié par trente-trois membres masculins de la famille des Taira, qui l'ont dédié aux divinités Shintô du Sanctuaire
d'Itsukushima «pour que soit exaucé leur vœu d'obtenir un meilleur sort dans le monde à venir» (II, p. 407). Ce texte fort intéressant fournit d'abord au lecteur, dans les trois premières parties, toutes les données requises pour lui permettre d'apprécier l'importance toute particulière que revêtaient les sûtras enluminés dans la culture religieuse de l'époque Heian (794-1185). Ces données sont particulièrement utiles au lecteur occidental peu familier avec la chronologie de l'histoire religieuse du Japon ancien et les grands noms qui en ont marqué l'évolution. La dernière partie du texte s'attache ensuite à décrire en détail le frontispice du premier rouleau, du rouleau 12 (chapitre du Don des Dieux), du rouleau 14 (chapitre de la Pratique commode) et du rouleau 27 (chapitre de la Conduite originelle du Roi Splendide), tout en fournissant des clés pour comprendre les scènes présentées. Des illustrations tirées de ces rouleaux facilitent la compréhension, bien que la qualité des reproductions en noir et blanc laisse à désirer.

En parallèle avec ces deux textes portant sur l'iconographie japonaise, on peut se replonger dans les représentations de l'au-delà dans l'iconographie chrétienne en lisant l'article de la professeure M. Fournié, de l'Université de Toulouse, sur le retable d'Enguerrand Quarton montrant Le couronnement de la Vierge (II, p. 325). Ce chef-d'œuvre du Moyen Âge, conservé au musée de Villeneuve-lès-Avignon en France, a été peint en 1454. L'article nous explique la structure de ce tableau et propose une analyse détaillée de ses divers éléments: le groupe central constitué par la Vierge couronnée par les trois personnes de la Trinité, la société des saints et des élus, le monde terrestre, le monde souterrain et les lieux de l'Au-delà, où l'on distingue le Paradis, l'Enfer et le Purgatoire ainsi que le Limbe des Enfants. Notons au passage que le lecteur trouvera une autre référence à l'iconographie chrétienne, cette fois-ci au sujet de la «bonne mort» comparée à la «mauvaise mort », à la fin de l'article de H. Shimauchi (II, p. 249) où il est brièvement fait référence à l'image du mois de décembre dans le Livre d'Heures de Jean Poyer (vers 1490).

\section{MALADIE ET GUÉRISON, FINITUDE ET SALUT}

Le professeur K. Tada nous présente ses recherches sur l'évolution survenue dans la conception de la maladie à mesure que le bouddhisme, prônant le concept de la rétribution, s'est diffusé dans la société de l'Antiquité (II, p. 312). L'auteur note qu'à l'époque ancienne au Japon «même si la maladie est un phénomène qui surgit dans le corps de l'individu, elle n'était pas un élément qui permette de questionner celui-ci à un niveau éthique» (II, p. 312). Or, selon le principe de rétribution, la maladie peut «se manifester comme le résultat d'une mauvaise action commise dans une vie précédente» (II, p. 313). Les extraits du Nihon Ryôiki et du Chinajiaibun cités dans l'article viennent utilement documenter cette évolution. Notons que le titre de ce deuxième texte signifie littéralement: Lamentation sur moi-même qui suis accablé par la maladie (II, p. 322). L'avant-dernière partie de l'article résume et explique - ce que plusieurs lecteurs occidentaux apprécieront - comment le bouddhisme, le confucianisme et le taoïsme ont négocié leur coexistence dans le Japon des six dynasties (II, p. 319-320).

Le lecteur qui s'intéresse en particulier au confucianisme voudra consulter l'article de G. Kurihara (II, p. 385) sur la conception de la vie et de la mort chez Jinsaï Ito (1627-1705). La conclusion de ce texte (traduit du japonais au français) contient les phrases suivantes qui méritent d'être citées, ne serait-ce que parce qu'elles expriment en des termes accessibles une interrogation sur le sens de la vie qui, lorsqu'elle fait référence à une philosophie orientale, est souvent présentée au lecteur occidental dans un vocabulaire trop exotique pour le rejoindre:

Jinsaï ne cesse de demander: "Comment un individu fini et seul peut-il surmonter sa finitude et participer à la vie infinie en tant que communauté s'ouvrant au monde entier et à tous les temps?» [...] Comment vivre? Quelle est la valeur de la vie ? Chez Jinsaï, ces questions ne proviennent pas du fait que tout homme est mortel, mais du fait qu'il existe un écart insurmontable entre le soi et I'autre. Quand Jinsaï parvient à trouver le moyen de dépasser cette distance, c'est-à-dire à saisir la vie comme la communauté entre le soi et l'autre en surmontant la vie finie du soi, autrement dit, quand il arrive à découvrir la vérité et la valeur de la vie au sein de la vie, il n'y a plus ni de place ni de nécessité de l'intervention de la mort. C'est alors que la vie infinie elle-même se révèle clairement, ce qui amène Jinsaï à la conclusion: "la vie existe, la mort n'existe pas " (p. 391; notre soulignement). 
La question du salut se pose avec une acuité troublante dans le cas des enfants. L'article de R. Ward (II, p. 367) se penche sur l'enseignement du bouddhisme de la Terre Pure, plus particulièrement de l'École Jõdo Shin, sur le sort des enfants dans l'au-delà. Le nœud du problème est lié au degré de conscience requis pour être sauvé: "if Shin fate is grounded in an active and cognizant realization of one's salvation, does this not exclude those who lack such a cognizance?» (II, p. 368). Le sort des enfants morts en bas âge, des enfants morts-nés et des fœtus inquiète les fidèles, ce qui a suscité l'émergence des pratiques dites du mizuko, qui ont pour but de contribuer à leur renaissance dans la Terre Pure, mais dont l'orthodoxie est contestée. Ward a su repérer, à partir du XVI ${ }^{\mathrm{e}}$ siècle, des sources peu connues, dit-il (II, p. 368), et il nous guide à l'intérieur du débat (p. 378, note 15) sur cette question angoissante pour beaucoup de parents, surtout lorsqu'il y a eu avortement.

Le thème de la maladie donne aussi lieu à une comparaison grâce à l'article préparé par le directeur de l'École française d'Extrême-Orient, sur les religions en Chine, F. Verellen, (I, p. 61). Notons que le titre de l'article ne fait pas référence à la maladie et à la finitude, mais bien plutôt à «la guérison et la rédemption». Le contexte étudié est celui de la Chine médiévale, plus précisément dans la tradition liturgique de la communauté dite des Maîtres célestes, une organisation ecclésiastique du taoïsme qui perdure de nos jours. Là encore, la maladie « est perçue comme un châtiment mérité en raison de transgressions morales ou de violations de tabou» (I, p. 62). Mais ce ne sont pas que les vivants qui sont malades, «les morts non apaisés sont source de miasmes et de contagions »; le rituel taoïste ancien de guérison, nous dit l'auteur, «s'attache ainsi à la rédemption des morts» (I, p. 62). L'article explique en détail le rituel de présentation des requêtes selon l'Almanach de requêtes du Maître Chisong, en insistant particulièrement sur la Requête de Pénitence en vue du destin personnel (I, p. 68), la Requête pour l'acquittement des inculpations (I, p. 72) et la Requête pour asperger la maison d'un nouveau défunt (I, p. 74). L'article se termine par une récapitulation en cinq points de la sotériologie des Maîtres célestes selon l'Allmanach des requêtes: 1) tout être humain meurt pécheur; 2) la mort est imaginée comme une incarcération dans l'obscurité où le nouveau défunt doit rendre compte de ses fautes; les morts nourrissent des ressentiments à l'encontre des vivants, y compris leurs parents; 3 ) les morts subissent aussi des détresses dues à de nouvelles infractions, par exemple la violation de leur tombeau; 4) le rituel de présentation de requêtes constitue le moyen d'obtenir l'acquittement et la libération des morts; et 5) les morts acquittés sont promus à une existence insouciante dans un hall de lumière (I, p. 75-76).

Également sur la Chine ancienne et médiévale, l'article de M. Ikezawa (II, p. 119) permet au lecteur de compléter ses connaissances sur «le salut apporté par les vivants aux morts en peine» (I, p. 129). L'auteur nous explique aussi comment le culte des ancêtres s'est modifié au contact du confucianisme (I, p. 125). Le titre de cet article le place sous le thème des "figures de compréhension de la "malemort" » plutôt que de la guérison. Signalons au passage la définition de ce terme, proposée dans l'article de A. Bouchy (I, p. 149-150) ainsi qu'en note dans l'avant-propos du volume I de La mort et les au-delàs: "mot ancien qui signifie littéralement la "mauvaise (male) mort" (ashiki shi), c'est-à-dire la mort tragique, cruelle, non "naturelle" ( (I, p. 9). Lors du colloque tenu à Tokyo, un atelier complet fut consacré au thème «Réagir face à la malemort», l'approche comparative étant dans ce cas mise à contribution de façon particulièrement frappante avec des présentations portant sur le Japon ( «a mort violente et son acceptation: Création d'une nouvelle "culture de la mort" », Namihira, I, p. 111); la Chine ("Exorcisme, rituels, reconnaissance, salvation», Ikezawa, I, p. 119), les Andes péruvienne («Entre récits de revenants et deuil suspendu », Robin Azevedo, I, p. 133). Un texte de synthèse de A. Bouchy, de l'École française d'Extrême-Orient, explore les recoupements entre ces trois cadres géographiques, historiques et sociaux. Dans son article, intitulé «Exclure, distinguer, relier: Le traitement de la malemort comme miroir de la gestion de soi, de l'"autre" et de la violence » (I, p. 149), elle s'attache à relever «les axes communs et les divergences », en adoptant comme point de vue l'anthropologie et l'ethnologie du Japon » (I, p. 149). On ne peut que chaleureusement recommander au lecteur ces textes sur le thème de la malemort, qui documentent les interrelations entre la survie de croyances anciennes et l'émergence d'une nouvelle culture de la mort en Amérique et en Asie.

On peut aussi rattacher à ce thème de la maladie et de la guérison un article où les pratiques documentées supposent que la personne reconnaît en elle les présages de la mort, qu'elle tente de s'y soustraire ou encore qu'elle accepte de ne pas guérir. Il s'agit du texte de T. Sugikie intitulé «Signes précurseurs de mort, esquive de mort et yoga de mort: Une scène de mort du haut Moyen Âge en Inde (I, p. 15).

\section{LES CONTACTS ENTRE LES MORTS ET LES VIVANTS}

Plusieurs sujets qui frappent l'imagination sont abordés sous le thème des contacts entre les morts et les vivants. Dans un article fort éclairant et bien référencé, le professeur Y. Ikegami, de l'Université de Komazawa, parle même "d'intimité des morts chez les Japonais ». Il en propose une analyse «sous l'angle de l'étude des croyances populaires» (I, p. 163). Il déclare, au début de son texte, qu'«il est indéniable qu'il existe dans la société japonaise des pratiques réputées permettre aux vivants d'agir directement sur les morts ou de communiquer avec eux.» (I, p. 163) et il discute, à la fin de l'article, de "l'avenir de cette "intimité des morts" dans le Japon contemporain » (I, p. 170-172). L'analyse fait ressortir les contrastes entre les cultures marquées par les enseignements des monothéismes et celles marquées par le bouddhisme en ce qui concerne les contacts directs et particuliers des vivants avec les morts et les pratiques visant soit à réprimer, soit à établir la communication mutuelle des uns avec les autres (I, p. 164). Pour illustrer son propos, l'auteur fait référence à une expérience de chamanisme dont il a été témoin, alors qu'il menait une enquête sur les croyances populaires dans la région de Tsugaru (I, p. 167). Il est utile de citer le passage suivant du texte car il donne au lecteur une perspective d'ensemble qui facilite la compréhension de plusieurs des éléments abordés dans d'autres articles portant sur le Japon dans les deux volumes de La mort et les au-delàs:

Les croyances populaires du Japon sont un exemple typique de structure souple dans la mesure où la manière d'agir auprès des morts consiste à raffiner des formes et des rites de pratique en maintenant la "foi » et les « concepts » dans un état ambigu; d'où son caractère secret lui permettant de survivre à travers les époques en conservant la présence intime des morts. II n'est donc pas exagéré de dire que les études modernes des croyances populaires ont pris une mauvaise direction dès le début parce qu'elles ont superposé les discours aux discours en vue d'éclaircir la "vision de l'au-delà chez les Japonais » ou la «vision de l'âme chez les Japonais» (I, p. 168).

Le texte ayant été traduit du japonais au français, il est impossible de savoir si la mention de "structure souple» fait effectivement référence ici à la méthodologie des systèmes souples (soft systems methodologies), mais c'est là une hypothèse qui pourrait ouvrir des perspectives inédites. Signalons pour le bénéfice des lecteurs qui voudraient lire un texte du professeur Ikegami portant plus spécifiquement sur le chamanisme au Japon que l'article Local Newspaper Coverage of Folk Shamans in Aomori Prefecture, publié dans la revue Folks Beliefs in Modern Japan, Contemporary Papers on Japanese Religion 3, 1994, p. 9-91, est accessible en ligne à l'adresse suivante: <www2.kokugakuin.ac.jp/ijcc/wp/cpjr/ folkbeliefs/ikegami.html>

Le chamanisme, sa position historique au Japon ainsi que sa place dans la vie des Japonais de nos jours nous sont aussi présentés avec des références à des études de terrain récentes dans un article du volume II de La mort et les au-delàs, intitulé: Les gens qui relient l'au-delà et l'ici-bas (Matsumoto, II, p. 219). Le phénomène des feux follets dans les traditions populaires donne lieu à des rapprochements entre les explications typiques données à ces apparitions tant au Japon qu'en Occident (Shimauchi, II, p. 237). Signalons que c'est parmi les notes de cet article que nous sont données quelques précisions utiles sur la notion de tama (II, p. 250, note 11), utilisée dans d'autres articles de La mort et les audelàs pour désigner l'âme.

La démarche comparative sur ce thème des contacts entre les morts et les vivants est particulièrement riche et nous amène à regrouper ici plusieurs articles répartis entre les deux volumes de La mort et les audelàs. Sur le phénomène de la possession, il y a d'abord le texte fouillé de C. Vassas sur Le Dibbuk, une forme de possession dans le judä̈sme ancien (I, p. 181). Nous passons ensuite à l'Inde et au jaïnisme, avec l'article de M. Carrin sur les phénomènes de possession induits lors de cérémonies religieuses, intitulé: Mourir pour devenir un demi-dieu: Les cultes des bhuta en pays Canara (I, p. 27).

Les références au monde chrétien nous sont proposées d'abord par A. Fine, du Centre d'anthropologie de Toulouse, dans un article sur Le parrain, son filleul, l'ici-bas et l'au-delà dans la société chrétienne (I, p. 173). La tradition chrétienne orthodoxe grecque est aussi évoquée 
grâce à un article de K. Seraïdari, du Centre d'anthropologie de Toulouse, intitulé: Usages funéraires dans la Grèce contemporaine (II, p. 393-405). L'auteure y présente une analyse détaillée d'une pratique qui suppose une communication entre les morts et les vivants: la fabrication par les femmes grecques des kolliva, ces pâtisseries qui sont ensuite bénies à l'église pour les défunts nommés par les femmes qui les ont confectionnés. "Selon une croyance populaire, les âmes des morts vivent dans un monde parallèle et passent un certain temps chaque année avec les vivants, qu'il faut chaque fois marquer par la fabrication des kolliva» (II, p. 394). L'auteur pose dès l'introduction que: "l'Église favorise l'image d'une communauté des morts, tandis que les femmes, qui cuisinent les kolliva, désirent rendre hommage à des défunts particuliers. La fabrication de ce gâteau entre dans la catégorie des tâches culinaires, dans la mesure où elle transpose l'obligation de préparer la nourriture pour la famille dans le domaine des morts. En transformant les cuisinières en médiatrices entre ce monde-ci et l'au-delà, cette coutume leur permet de créer des liens entre elles, de réinterpréter la doctrine ecclésiastique et d'accomplir leur parcours féminin » (II, p. 394). Le passage suivant, tiré de la conclusion, est particulièrement éloquent sur l'intensité des contacts entre les morts et les vivants: "Comme ma grand-mère paternelle me l'a expliqué, "si tu veux, tu peux commémorer quelqu'un qui est mort il y a deux siècles; car le mort ne vieillit pas" [...] Avec leurs listes qui établissent des priorités et qui concrétisent leurs affinités, les femmes mettent en ordre les morts; ce sont elles aussi qui les regroupent et les singularisent, qui s'approprient leur pouvoir et allègent leur souffrance. La confection des kolliva [...] c'est un geste d'amour, adressé à des personnes désignées par leur nom, qui matérialise le partage d'une même nourriture entre vivants et morts, et qui réintègre les défunts dans l'espace domestique» (II, p. 404).

À l'opposé, pourrions-nous évoquer le refus d'un contact trop intense avec les morts pour expliquer l'évolution des sensibilités en Occident lorsqu'il s'agit de choisir quels espaces deviendront des cimetières, comment ils seront aménagés et comment ils se modifieront avec la popularité grandissante de la crémation au détriment de l'inhumation de la dépouille? Ce sont de telles questions qui viennent à l'esprit à la lecture du texte bien documenté de C. Armanet, de l'Université Lille I, sur la sépulture cinéraire en France (II, 209). L'auteure y dresse un état de la situation, couvrant à la fois l'historique de la crémation en France et les diverses formes de sépultures de cendres.

\section{ROMANS, BANDES DESSINÉES ET THÉÂTRE}

Trois textes ouvrent des fenêtres inédites en se servant du roman, de la bande dessinée et du théâtre pour explorer comment les croyances traditionnelles sur l'au-delà ont été transposées dans la culture du Japon moderne. L'article de K. Date (II, p. 293) nous présente le parcours individuel et l'œuvre d'un écrivain japonais du XX $\mathrm{XX}^{\mathrm{e}}$ siècle, Kunio Tsuji (1925-1999) et analyse les rapports entre la vie et la mort dans trois de ses romans. On sait que la bande dessinée (le manga) occupe une place importante dans la culture japonaise contemporaine et on constate avec plaisir qu'un article, où figurent quelques illustrations, nous présente une bande dessinée de Shiriagari Kotobuki intitulée Yaji et Kita de minuit (Fukushima, II, p. 281). Les deux héros de cette bande dessinée, motivés par leur désir d'échapper aux drogues, se rendent en pèlerinage au sanctuaire d'Ise. Dans son analyse, un peu touffue par ailleurs, l'auteur de l'article retrace les liens entre les péripéties des personnages de cette bande dessinée et la croyance, héritée de la tradition, qui veut que toute personne ait son "double». L'être mort et son double agissent dans l'histoire, sans que le lecteur puisse faire la part entre le réel et le songe. Les personnages eux-mêmes choisissent de ne pas lever l'équivoque (II, p. 289), une décision qui semble rencontrer une certaine approbation chez le lecteur contemporain, si on en juge par la popularité de la bande dessinée.

Le théâtre se révèle aussi un lieu où la célébration de la mémoire des morts selon le diktat de la tradition survit sous une autre forme. C. Sato (II, p. 271) nous guide dans l'Histoire de Soga, une sorte de chronique militaire qui a été théâtralisée dès le Moyen Âge et dont certaines scènes font encore partie aujourd'hui du répertoire du Kabuki (II, p. 279).

La démarche comparative est possible, ici encore, grâce à un article sur le ballet Giselle, intitulé: D'un "esprit sans corps » au "corps sans esprit». Approche des représentations de la mort dans le ballet Giselle (Fournié, D., II, p. 257).

\section{MORT VOLONTAIRE}

On ne saurait passer sous silence la remarquable contribution du professeur Jean-Pierre Albert, directeur du Centre d'anthropologie de Toulouse, dont l'article s'intitule: Martyre et mort volontaire en Europe. Entre religion et politique (I, p. 79). L'auteur y discute de façon nuancée les deux figures de mort sinon volontaire, du moins consentie, qui suscitent des éloges: le martyre à motivation religieuse et le fait de «mourir pour la Patrie » (I, p. 81). L'hypothèse développée « consiste à tenir les formes et le degré de reconnaissance de la mort volontaire comme un bon indicateur du degré de holisme et d'individualisme caractéristique des différentes sociétés» (I, p. 90). Dans sa conclusion, l'auteur signale un phénomène qui, selon nous, n'attire pas suffisamment l'attention de nos contemporains : l'apparition dans nos sociétés d'une

[...] nouvelle sorte de héros exemplaires, faisant l'objet d'une reconnaissance massive [...] II s'agit principalement d'auteurs d'exploits dans les registres du sport et de l'aventure [...]. En fait, ce qui "force le respect» dans de tels exemples est moins la valeur intrinsèque du but poursuivi que le mérite reconnu à la ténacité, la fidélité à soi-même, la capacité de vivre jusqu'au bout un idéal que l'on a choisi. [...] [L]a nouveauté de la situation présente tient à la valorisation à peu près exclusive de cette dimension subjective, ou formelle de l'héroïsme: c'est elle qui est considérée comme la valeur humaine par excellence et, en conséquence, le héros souffrant devient un exemplaire remarquable de l'humanité (I, p. 91).

Signalons, pour faire suite au texte du professeur Albert, l'article (en anglais) de M. Fukuoka (II, p. 340) qui porte à notre attention un cas de mort volontaire appelée au Japon junshi, qui consiste à suivre l'empereur dans la mort. C'est ce qu'ont choisi de faire le général Nogi Maresuke et son épouse en 1912, pendant que se déroulaient les funérailles de l'empereur Meiji. Le général avait pris les dispositions nécessaires pour qu'un photographe se présente chez lui, le matin du jour où eut lieu ce double suicide. L'article contient une reproduction des deux photos, celle du général et celle de son épouse; ce sont ces photos ainsi que les circonstances qui ont entouré leur exécution qui guident l'auteur de l'article dans son analyse.

\section{LA MORT EN CLASSE}

Deux articles qui n'ont pu être présentés sous les thèmes choisis jusqu'ici nous mettent en présence de la mort dans une salle de classe: avec des jeunes enfants et avec des étudiants universitaires. Le texte de N. Nijima prend l'exemple de l'attitude des jeunes enfants envers les animaux à l'école pour examiner les rapports qu'entretiennent les Japonais entre la vie et la mort (II, p. 197). Le texte de E. Godeau, médecin, nous décrit l'expérience vécue par des étudiants universitaires en classe de dissection (II, p. 349; en anglais, avec photographies prises par l'auteure à Toulouse).

\section{SITES INTERNET}

Il faut souhaiter que les travaux de ces chercheurs sur la mort et les au-delàs puissent connaître une vaste diffusion et que d'autres projets de collaborations interdisciplinaires et interculturelles viennent élargir encore l'éventail des thèmes abordés. Les sites Internet des deux organismes qui ont collaboré à ce projet, à Toulouse et à Tokyo, nous permettront probablement de connaître la suite des travaux. Les voici:

Le site du 21st Century COE Program, Death and Life Studies se trouve à l'adresse suivante: <www.l.u-tokyo.ac.jp/shiseigaku/en_index. $\mathrm{html}>$. On y trouve la table des matières du volume I de La mort et les au-delàs à l'adresse: <www.l.u-tokyo.ac.jp/shiseigaku/en/publication/ seika_bull3.htm>.

Le site du Centre d'anthropologie de Toulouse se trouve à l'adresse : $<$ www.ethno-info.com/index.php?id=33>. La page consacrée à l'ethnologie du Japon se trouve à l'adresse: <www.ethno-info.com/index. $\mathrm{php}$ ? $\mathrm{id}=37>$. On y trouve le programme du colloque tenu à Toulouse en octobre 2006 ainsi que le résumé en français des interventions des membres du Centre d'anthropologie de Toulouse lors du colloque tenu à Tokyo en février 2006. Signalons que ces données n'apparaissent pas immédiatement à l'écran et qu'il faut poursuivre la lecture en faisant dérouler le texte pour y avoir accès.

Pour conclure, on ne saurait trop insister sur l'importance de la perspective interdisciplinaire pour une étude féconde des thèmes qui se rattachent à la mort et à l'au-delà. Ces deux publications de l'Université de Tokyo en font une démonstration convaincante. 